\title{
On-chip surface-enhanced Raman scattering detection using integrated liquid-core waveguides
}

\author{
Aaron R. Hawkins \\ hawkins@ee.byu.edu \\ Evan J. Lunt \\ Phillip Measor \\ Leo Seballos \\ Dongliang Yin
}

See next page for additional authors

Follow this and additional works at: https://scholarsarchive.byu.edu/facpub

Part of the Electrical and Computer Engineering Commons

\section{Original Publication Citation}

Measor, Philip, Leo Seballos, Dongliang Yin, Jin Z. Zhang, Evan J. Lunt, Aaron R. Hawkins, and Holger Schmidt. "On-chip surface-enhanced Raman scattering detection using integrated liquidcore waveguides." Applied Physics Letters 9 (27)

\section{BYU ScholarsArchive Citation}

Hawkins, Aaron R.; Lunt, Evan J.; Measor, Phillip; Seballos, Leo; Yin, Dongliang; Zhang, Jin Z.; and Schmidt, Holger, "On-chip surface-enhanced Raman scattering detection using integrated liquid-core waveguides" (2007). Faculty Publications. 255.

https://scholarsarchive.byu.edu/facpub/255

This Peer-Reviewed Article is brought to you for free and open access by BYU ScholarsArchive. It has been accepted for inclusion in Faculty Publications by an authorized administrator of BYU ScholarsArchive. For more information, please contact ellen_amatangelo@byu.edu. 
Authors

Aaron R. Hawkins, Evan J. Lunt, Phillip Measor, Leo Seballos, Dongliang Yin, Jin Z. Zhang, and Holger Schmidt 


\title{
On-chip surface-enhanced Raman scattering detection using integrated liquid-core waveguides
}

\author{
Philip Measor, ${ }^{\text {a) }}$ Leo Seballos, Dongliang Yin, and Jin Z. Zhang \\ School of Engineering and Department of Chemistry and Biochemistry, University of California Santa Cruz, \\ 1156 High Street, Santa Cruz, California 95064 \\ Evan J. Lunt and Aaron R. Hawkins \\ Department of Electrical and Computer Engineering, Brigham Young University, 459 Clyde Building, \\ Provo, Utah 84604 \\ Holger Schmidt \\ School of Engineering, University of California Santa Cruz, 1156 High Street, Santa Cruz, California 95064
}

(Received 18 April 2007; accepted 27 April 2007; published online 21 May 2007)

\begin{abstract}
The authors demonstrate surface-enhanced Raman scattering (SERS) detection on an optofluidic chip. Interconnected solid- and liquid-core antiresonant reflecting optical waveguides (ARROWs) form a planar beam geometry that allows for high mode intensities along microfluidic channels containing molecules optimized for SERS. The excitation power and concentration dependence of SERS from rhodamine $6 \mathrm{G}$ (R6G) molecules adsorbed to silver nanoparticles were systematically studied. The data can be described by a model that takes into account the microphotonic structure. Detection sensitivity to a minimum concentration of $30 \mathrm{nM}$ is found, demonstrating the suitability of ARROW-based optofluidic chips for high sensitivity detection with molecular specificity. (C) 2007 American Institute of Physics. [DOI: 10.1063/1.2742287]
\end{abstract}

Molecular detection devices are of great interest for many fields with a plethora of applications including cancer detection, DNA sequencing, infectious disease detection, and basic research. Raman sensing based on inelastic light scattering is particularly attractive due to its molecular sensitivity. Its main disadvantage is the intrinsically low Raman cross section, but when a molecule is adsorbed to a metallic nanoparticle, small compared to the wavelength of light, the molecule's Raman cross section can be greatly increased due to electric field enhancement on the nanoparticle surface. Surface-enhanced Raman scattering (SERS) techniques have been used for over 30 years ${ }^{1-3}$ for chemical and biosensing applications ${ }^{4}$ and recently for single molecule detection., ${ }^{5,6}$ Typical SERS detection schemes use bulky microscope setups with discrete optical elements at high costs. ${ }^{7}$ Thus, an integrated optics approach to SERS would be desirable. Recently, several methods using liquid-core fibers, ${ }^{8}$ D-shaped fibers, ${ }^{9}$ and photonic crystal fibers ${ }^{10}$ have been demonstrated but are difficult to integrate. Liquid-core antiresonant reflecting optical waveguides ${ }^{11,12}$ (ARROWs) are particularly attractive because they can provide high excitation intensities over long distances and offer fully planar beam geometries when interfaced with solid-core waveguides. ${ }^{13}$ Highly efficient fluorescence detection ${ }^{14}$ down to single molecule sensitivity has been demonstrated ${ }^{15}$ but without the rich molecule-specific information that SERS provides. In this letter, we demonstrate SER detection from an on-chip liquidcore ARROW waveguide in planar beam geometry. We determine the excitation power and concentration dependence of SERS from rhodamine $6 \mathrm{G}$ (R6G) molecules as well as the sensitivity limit and steps for further optimization.

Planar integrated liquid-core ARROW waveguides consisting of a $\mathrm{Si}$ substrate with alternating $\mathrm{SiN}$ and $\mathrm{SiO}_{2}$ dielec-

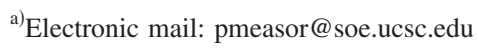

tric layers surrounding a liquid core ${ }^{11}[$ see Fig. 1(a)] are produced with standard silicon microfabrication techniques in a sacrificial layer process. ${ }^{16}$ The ARROW used in this study had a $12 \mu \mathrm{m}$ wide by $5 \mu \mathrm{m}$ high hollow core and cladding thicknesses of 100/268 and 120/337 nm for the bottom and top thin $\mathrm{SiN} / \mathrm{SiO}_{2}$ layers, respectively. The top thick $\mathrm{SiO}_{2}$ layer had a thickness of $2.476 \mu \mathrm{m}$. The liquid-core ARROW SERS detection setup, as shown on Fig. 1(b), consists of a helium neon $(\mathrm{HeNe})$ laser $\left(\lambda_{\mathrm{exc}}=632.8 \mathrm{~nm}\right)$ source which
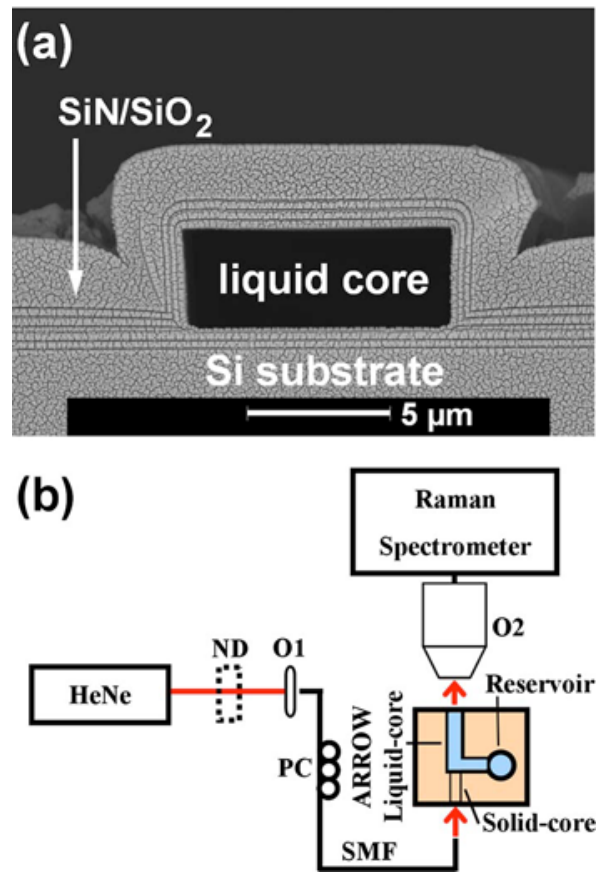

FIG. 1. (Color online) (a) ARROW waveguide cross section consisting of $\mathrm{SiN}$ and $\mathrm{SiO}_{2}$ dielectric layers and a liquid core (Ref. 11). (b) Experimental setup. 


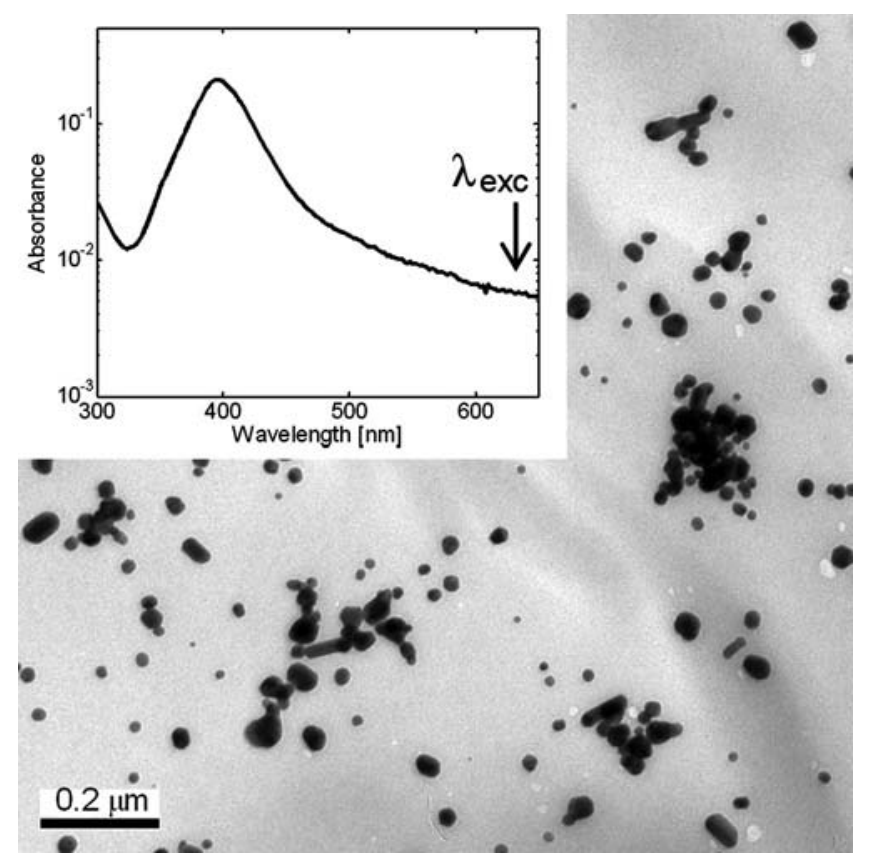

FIG. 2. TEM image of silver nanoparticles used in the study showing a narrow size distribution of $20 \mathrm{~nm}$ average diameter spherical nanoparticles with an $11 \mathrm{~nm}$ variance. Inset: Corrresponding UV-vis absorption spectra of nanoparticles in solution.

propagates through neutral density filters for the power dependence measurements. The beam is coupled via objective O1 into a single-mode fiber which is wound through a polarization controller and aligned to the ARROW. The liquidcore ARROW platform used in this study was an advancement over straight liquid-core ARROWs (Refs. 11-15) (where no solid core or reservoir existed), consisting of a solid-core waveguide $(12 \mu \mathrm{m}$ wide, $2.5 \mu \mathrm{m}$ high, and $1.1 \mu \mathrm{m}$ etch depth) fabricated and aligned to the liquid-core ARROW, as shown in Fig. 1(b). A $10 \mu$ on-chip reservoir is depicted, into which the solution was pipetted and subsequently filled the core preventing solution evaporation during measurements. Finally, the output light is collected with a $100 \times 0.85$ numerical aperture objective lens $(\mathrm{O} 2)$ attached to a Raman spectrometer (Jobin Yvon). The SERS samples for this study consisted of solutions of varying concentrations (nominally $0.3,1,3,10$, or $30 \mu \mathrm{M}$ ) of R6G molecules and sodium chloride-induced aggregated silver nanoparticles $(1 \mathrm{nM})$. This system has been extensively studied providing a good proof-of-concept system for SERS detection experiments. ${ }^{17}$ The solutions were prepared by mixing an aqueous R6G solution $(18 \mathrm{M} \Omega \mathrm{cm}$ water or ethanol or ethanol/water) with silver nanoparticles that were synthesized using a citrate reducing agent. ${ }^{18}$ The solution was incubated for $30 \mathrm{~min}$ at room temperature, then activated with a sodium chloride $(\mathrm{NaCl})$ solution. The final $\mathrm{NaCl}$ concentration of all samples was $2.5 \mathrm{mM}$. Immediately after salt introduction, the solution was pipetted into the reservoir for measurements. Transmission electron microscopy (TEM) images [Fig. 2(a)] showed the mostly spherical nanoparticle consistency with a mean particle diameter of $20 \mathrm{~nm}$ and a standard deviation of $11 \mathrm{~nm}$. UV-vis spectra (Fig. 2 inset) showed a narrow resonance at $400 \mathrm{~nm}$ and some absorbance at the excitation wavelength $(632.8 \mathrm{~nm})$ indicating low heat generation due to absorption. The relatively narrow resoDownloaded 10 Feb 2009 to 128.187.0.164. Redistribution subject

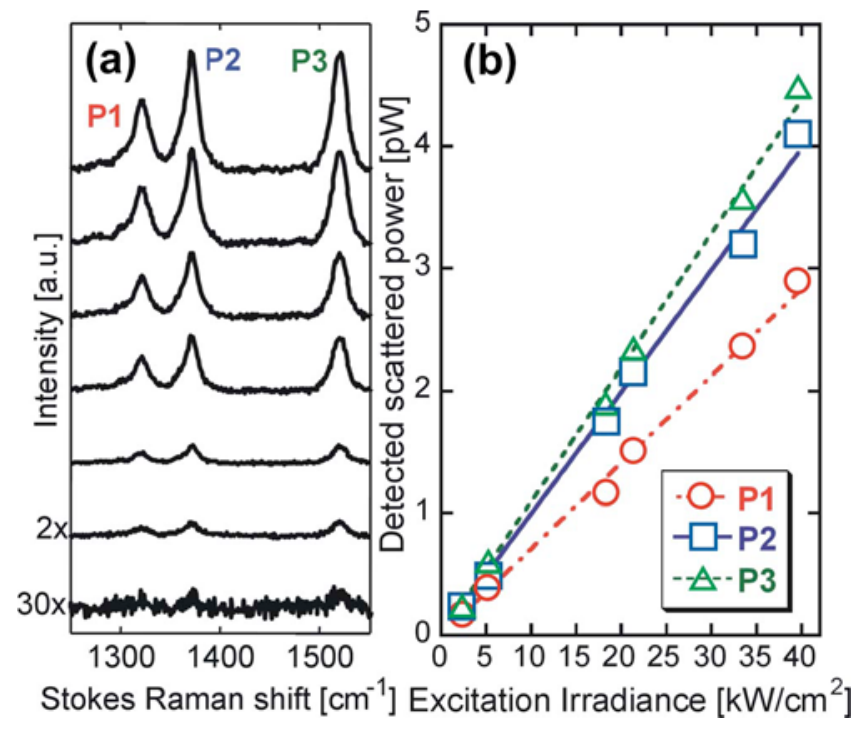

FIG. 3. (Color online) (a) Power dependence of detected SERS signals at $300 \mathrm{nM}$ active R6G concentration. The two lowest power signals are multiplied by factors of 2 and 30 as denoted. (b) Symbols: detected SERS power vs excitation irradiance. Lines: theory fits with Eq. (1).

nance peak is consistent with the homogeneity seen in the TEMs.

The detected SERS time-averaged spectra are plotted in Fig. 3(a) over the range of excitation input irradiances of $2-40 \mathrm{~kW} / \mathrm{cm}^{2}$, showing the characteristic R6G vibrational peaks with Stokes-shifted wave numbers of approximately 1320,1370 , and $1520 \mathrm{~cm}^{-1}$ (corresponding to the strong $\mathrm{C}-\mathrm{O}-\mathrm{C}$ and aromatic $\mathrm{C}-\mathrm{C}$ stretch vibrations). ${ }^{17}$ The points on Fig. 3(b) represent the area under these Raman peaks after background subtraction for a nominal R6G concentration of $3 \mu \mathrm{M}$ obtained from Gaussian-Lorentzian fits. The detected spontaneous Stokes-shifted Raman scattered power $P_{\mathrm{ds}}$ generated in the ARROW chip can be expressed by

$$
P_{\mathrm{ds}}=F_{\text {coll }} \frac{\lambda_{e}}{\lambda_{s}} \kappa e^{-\alpha_{1} L_{1}} \frac{\left(1-e^{-\alpha_{2} L_{2}}\right)}{\alpha_{2} L_{2}} N^{2} \sigma_{\mathrm{SERS}} I_{i},
$$

with the following defined as the angular collection factor $F_{\text {coll }}$, excitation light wavelength $\lambda_{e}$, Stokes-shifted scattered light wavelength $\lambda_{s}$, device coupling coefficient $\kappa$, solid-core waveguide loss $\alpha_{1}$ and length $L_{1}$, ARROW core loss $\alpha_{2}$ and length $L_{2}$, number of adsorbed molecules $N$ (which may be less than the number of molecules in solution), effective SERS Raman cross section $\sigma_{\text {SERS }}$, and input excitation irradiance $I_{i}$. This formula assumes negligible sample absorption and stimulated Raman scattering, identical waveguide losses for the excitation and scattered light, and a quadratic dependence of the number of adsorbed R6G molecules, as discussed below (see Fig. 4). Fits to the data using Eq. (1) are shown as lines in Fig. 3(b) and show very good agreement with $\kappa N^{2}$ as the only free fitting parameter. Independently measured values were used for all other terms such as the angular collection factor, ${ }^{14}$ liquid-core ARROW loss, ${ }^{14}$ and solid-core loss. ${ }^{13}$ The SERS cross sections $\sigma_{\text {SERS }}$ were obtained from bulk microscopy measurements of a drop of solution and showed a relatively low enhancement factor of $10^{2}-10^{3}$. Differences in frequency and polarizability for each vibrational mode result in different values for $\sigma_{\text {SERS }}$ and account for the different slopes observed in Fig. 3(b).

to AlP license or copyright; see http://apl.aip.org/apl/copyright.jsp 

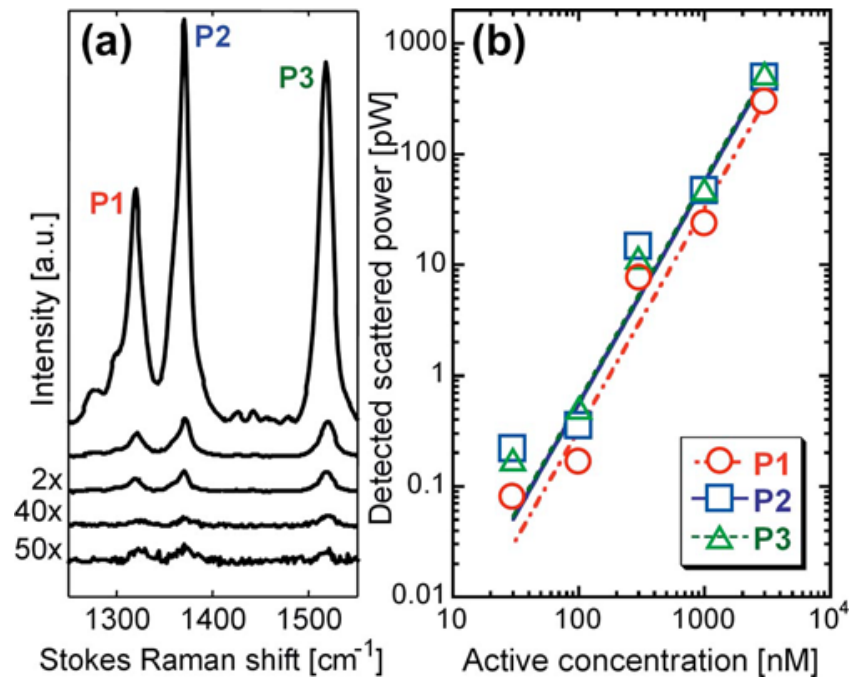

FIG. 4. (Color online) (a) Detected SERS signals at different active concentrations corrected to a nominal input excitation irradiance level of $15.2 \mathrm{~kW} / \mathrm{cm}^{2}$ for differing alignments between measurements. The lowest three concentrations are multiplied by factors of 2, 40, and 50 as denoted. (b) Symbols: detected SERS power vs concentration. Lines: quadratic fits with Eq. (1).

In order to determine the sensitivity limit of ARROWbased SERS detection, SERS spectra for different R6G concentrations were taken and are shown in Fig. 4(a). The concentration dependent data were taken from the same waveguide starting with the lowest R6G concentration. After each measurement, the solution was then suctioned out via the reservoir, and the waveguide was flushed with water before higher concentration solutions were added and tested. This was done to avoid false results due to contamination of the channels with residual molecules. The results shown on Fig. 4(b) were accumulated in a similar way as the power measurements discussed above except that the output signal was adjusted to a nominal excitation light output level to correct for the differing alignments between measurements. The spectra were collected for a nominal R6G concentration range of $300 \mathrm{nM}-30 \mu \mathrm{M}$. Generally, one expects a change of the SERS signal with increasing R6G concentration from linear to quadratic to saturation. This dependence is due to the increasing coverage of the nanoparticles until all binding sites are occupied. ${ }^{19}$ The SERS signals from the ARROW chip show a quadratic dependence at all but the lowest concentrations as is evident by the very good agreement with parabolic fits (lines). At the lowest concentration, the power dependence becomes linear. Control measurements with bulk microscopy using the same nominal concentrations (not shown), however, showed a quadratic dependence at the lowest concentrations and saturation at the highest concentrations. By comparing the concentration dependence of the ARROW and bulk measurements, we conclude that the $a c$ tive concentration of R6G molecules in the ARROW is approximately ten times lower than the nominal concentration. We attribute this difference to R6G adsorption on the ARROW's SiN walls which could be prevented by a suitable pretreatment of the walls. Therefore, the concentration dependent data are plotted as a function of active R6G concentration in Fig. 4(b). The minimum detected active R6G concentration of $30 \mathrm{nM}$ is almost three orders of magnitude lower than previous demonstrations using a photonic crystal fiber, ${ }^{10}$ and corresponds to detecting approximately $4 \times 10^{5}$ molecules in the ARROW excitation volume of $44 \mathrm{pl}$. This concentration limit is comparable to the reported $10 \mathrm{nM}$ limit in a liquid-core fiber $^{8}$ for a solution volume of $3 \mu \mathrm{l}$ with nanoparticle resonance excitation.

The presented measurements show the suitability of liquid-core ARROW waveguide structures for highly sensitive SERS detection with molecular specificity. Its main advantages are the unification of optical and fluidic paths, the high excitation intensities afforded by the micron-scale quasi-single-mode waveguides, and the ability to build a planar optofluidic infrastructure of interconnected solid- and hollow-core waveguides. SERS detection with nanomolar sensitivity was demonstrated. This limit can be further improved by utilization of resonance SERS detection ${ }^{19,20}$ or use of $\mathrm{Ag}$ or $\mathrm{Au}$ nanostructures ${ }^{21}$ which result in larger enhancements of the Raman cross section. These, combined with further optimization of the ARROW platform including reduced losses, use of waveguide intersections to define subpicoliter excitation volumes, ${ }^{15}$ and integration of wavelength filtering ${ }^{13}$ will push ARROW-based SERS detection towards the single molecule detection limit.

The authors acknowledge helpful discussions with S. Kühn, use of Raman spectrometer from A. Shakouri, and financial support from the National Academies Keck Futures Initiative under Grant No. NAKFI-Nano14, the National Science Foundation under Grant No. ECS-0528730, and the NIH/NIBIB under Grant No. 5-RO1-EB006097.

${ }^{1}$ D. L. Jeanmaire and R. P. Van Duyne, J. Electroanal. Chem. Interfacial Electrochem. 84, 1 (1977).

${ }^{2}$ M. Moskovits, Rev. Mod. Phys. 57, 783 (1985).

${ }^{3}$ A. Otto, I. Mrozek, H. Grabhorn, and W. Akemann, J. Phys.: Condens. Matter 4, 1143 (1992).

${ }^{4}$ K. Kneipp, H. Kneipp, I. Itzkan, R. R. Dasari, and M. S. Feld, J. Phys.: Condens. Matter 14, R597 (2002).

${ }^{5}$ S. Nie and S. R. Emory, Science 275, 1102 (1997).

${ }^{6}$ K. Kneipp, Y. Wang, H. Kneipp, L. T. Perelman, I. Itzkan, R. R. Dasari, and M. S. Feld, Phys. Rev. Lett. 78, 1667 (1997).

${ }^{7}$ M. B. Wabuyele, F. Yan, G. D. Griffin, and T. Vo-Dinh, Rev. Sci. Instrum. 76, 063710 (2005).

${ }^{8}$ W. Xu, S. Xu, Z. Lü, L. Chen, B. Zhao, and Y. Ozaki, Appl. Spectrosc. 58, 414 (2004).

${ }^{9}$ Y. Zhang, C. Gu, A. M. Schwartzberg, and J. Z. Zhang, Appl. Phys. Lett. 87, 123105 (2005).

${ }^{10}$ H. Yan, C. Gu, C. Yang, J. Liu, G. Jin, Jiatao Zhang, L. Hou, and Y. Yao, Appl. Phys. Lett. 89, 204101 (2006).

${ }^{11}$ D. Yin, J. P. Barber, A. R. Hawkins, D. W. Deamer, and H. Schmidt, Appl. Phys. Lett. 85, 3477 (2004).

${ }^{12}$ S. Campopiano, R. Bernini, L. Zeni, and P. M. Sarro, Opt. Lett. 29, 1894 (2004).

${ }^{13}$ H. Schmidt, D. Yin, J. P. Barber, and A. R. Hawkins, IEEE J. Sel. Top. Quantum Electron. 11, 519 (2005).

${ }^{14}$ D. Yin, J. P. Barber, A. R. Hawkins, and H. Schmidt, Appl. Phys. Lett. 87, 211111 (2005).

${ }^{15}$ D. Yin, J. P. Barber, D. W. Deamer, A. R. Hawkins, and H. Schmidt, Opt. Lett. 31, 2136 (2006).

${ }^{16}$ J. P. Barber, D. B. Conkey, J. R. Lee, N. B. Hubbard, L. L. Howell, D. Yin, H. Schmidt, and A. R. Hawkins, IEEE Photonics Technol. Lett. 17, 363 (2005).

${ }^{17}$ P. Hildebrandt and M. Stockburger, J. Phys. Chem. 88, 5935 (1984).

${ }^{18}$ P. C. Lee and D. Meisel, J. Phys. Chem. 86, 3391 (1982).

${ }^{19}$ C. McLaughlin, D. Graham, and W. E. Smith, J. Phys. Chem. B 106, 5408 (2002).

${ }^{20}$ O. Silman, A. Lepp, and M. Kerker, J. Phys. Chem. 87, 5319 (1983).

${ }^{21}$ A. M. Schwartzberg, C. D. Grant, A. Wolcott, C. E. Talley, T. R. Huser, R. Bogomolni, and J. Z. Zhang, J. Phys. Chem. B 108, 19191 (2004). 